\title{
GRAVITATIONAL WAVES FROM ECCENTRIC INTERMEDIATE-MASS BLACK HOLE BINARIES
}

\author{
Pau Amaro-Seoane ${ }^{1,2}$, M. Coleman Miller ${ }^{3}$, and Marc Freitag $^{4}$ \\ ${ }^{1}$ Institut de Ciències de l'Espai, IEEC/CSIC, Campus UAB, Torre C-5, parells, $2^{\text {na }}$ planta, ES-08193, Bellaterra, Barcelona, Spain \\ ${ }^{2}$ Max Planck Institut für Gravitationsphysik (Albert-Einstein-Institut), Am Mühlenberg 1, D-14476 Potsdam, Germany; Pau.Amaro-Seoane@aei.mpg.de \\ ${ }^{3}$ Department of Astronomy and Maryland Astronomy Center for Theory and Computation, University of Maryland, College Park, MD 20742-2421, USA; \\ miller@astro.umd.edu \\ ${ }^{4}$ Institute of Astronomy, University of Cambridge, Madingley Road, CB3 0HA Cambridge, UK; freitag@ast.cam.ac.uk \\ Received 2008 June 30; accepted 2008 December 26; published 2009 January 22
}

\begin{abstract}
If binary intermediate-mass black holes (IMBHs; with masses between 100 and $10^{4} M_{\odot}$ ) form in dense stellar clusters, their inspiral will be detectable with the planned Laser Interferometer Space Antenna (LISA) out to several Gpc. Here, we present a study of the dynamical evolution of such binaries using a combination of direct $N$-body techniques (when the binaries are well separated) and three-body relativistic scattering experiments (when the binaries are tight enough that interactions with stars occur one at a time). We find that for reasonable IMBH masses there is only a mild effect on the structure of the surrounding cluster even though the binary binding energy can exceed the binding energy of the cluster. We demonstrate that, contrary to standard assumptions, the eccentricity in the LISA band can be in some cases as large as $\sim 0.2-0.3$ and that it induces a measurable phase difference from circular binaries in the last year before merger. We also show that, even though energy input from the binary decreases the density of the core and slows down interactions, the total time to coalescence is short enough (typically less than a 100 million years) that such mergers will be unique snapshots of clustered star formation.
\end{abstract}

Key words: black hole physics - gravitational waves - methods: N-body simulations - stellar dynamics

\section{INTRODUCTION}

The existence of intermediate-mass black holes (IMBHs; masses $M \sim 10^{2-4} M_{\odot}$ ) is not as certain as that of stellar-mass or supermassive black holes because there is as yet no conclusively established dynamical mass for any candidate, although there is strong circumstantial evidence for this mass range in several cases (see Miller \& Colbert 2004, and references therein, for a review). Mergers of IMBHs would, however, be strong sources of gravitational waves.

The best studied scenario is the runaway growth of a star in a young cluster via physical collisions among the most massive stars in the center, which have sunk through mass segregation (Portegies Zwart \& McMillan 2000; Gürkan et al. 2004; Portegies Zwart et al. 2004; Freitag et al. 2006). Recently, Gürkan et al. (2006) addressed the same configuration but added a fraction of primordial binaries to the stellar system. Using a Monte Carlo stellar-dynamics code, they found that not one but two very massive stars grow in rich clusters in which $10 \%$ or more of stars are in primordial hard binaries, suggesting the formation of two IMBHs. However, this result has not been confirmed yet using more accurate direct $N$-body simulations. Portegies Zwart et al. (2004) have a simulation with primordial binaries but they do not see this formation, though it is also currently unclear how different core concentrations will affect binary IMBH formation with a certain fraction of primordial binaries. It is also possible that wind losses may drive away mass more rapidly than it accretes through further collisions (see Belkus et al. 2007), although this relies on uncertain extrapolations from the $\sim 120 M_{\odot}$ that is the top of their range (see their Table 2 ) to the $\sim 2000 M_{\odot}$ masses observed in $N$-body simulations.

Fregeau et al. (2006) considered for the first time the possibility that such a binary could be observed thanks to the emission of gravitational waves in the coalescence phase and estimated that one can expect the Laser Interferometer Space Antenna (LISA) to detect tens of them depending on the distribution of cluster masses and densities. Amaro-Seoane \& Freitag (2006) addressed the evolution of a binary of two IMBHs formed as the result of the collision of two independent stellar clusters and followed the parameters of the binary orbit down to the region in which it will emit gravitational waves in the $\sim 10^{-4} \mathrm{~Hz}-10^{-1} \mathrm{~Hz}$ LISA domain. To do this, they combined direct-summation simulations with an analytical model to evolve the binary from a point in which it was hard.

Here we assume that an IMBH binary has been produced in a single dense stellar cluster, and study the subsequent sinking of the IMBHs and the evolution and properties of the binary when it forms. In Section 2, we discuss our numerical method, which combines direct $N$-body studies with three-body scattering integrations. In Section 3, we discuss the astrophysical implications of our results.

\section{EVOLUTION OF THE INTERMEDIATE-MASS BLACK HOLE PAIR: NUMERICAL METHOD}

\subsection{Direct $N$-body Simulations}

Direct $N$-body codes integrate all gravitational accelerations in a stellar system without supposing any special symmetries. They are thus the most general and robust tools for numerical analysis of stellar clusters (Aarseth 1999, 2003). The code we use, NBODY4, includes a variety of sophisticated approaches that improve speed and accuracy, including KS regularization (Kustaanheimo \& Stiefel 1965), as well as triple (three-body subsystems), quad (four-body subsystems), and chain regularization (Aarseth 1999, 2003). It also does not make use of any softening, which would lead to unrealistic evolution of the orbital parameters of the binary of massive black holes. The disadvantage of this or any direct $N$-body code is the required computational time. However, our calculations are accelerated thanks to the special-purpose hardware GRAPE-6A single PCI cards of the AEI cluster TUFFSTEIN used for the simulations. 


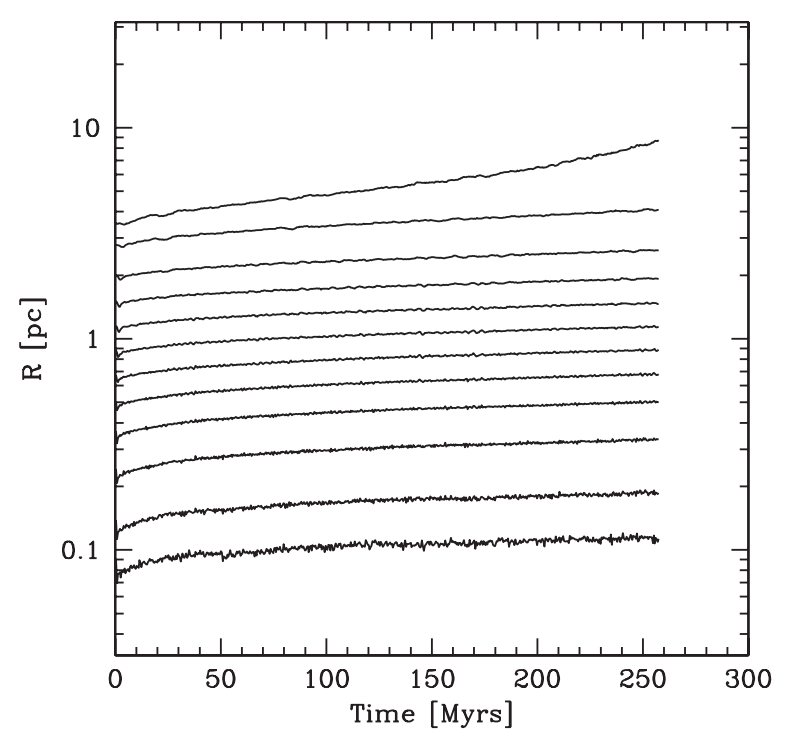

Figure 1. Lagrangian radii showing the evolution of different mass fractions in the cluster for Model D. The fractions are, from the bottom to the top, $0.01 \%$, $0.03 \%, 0.1 \%, 0.2 \%, 0.3 \%, \ldots, 0.9 \%$, and $0.95 \%$.

Table 1

Initial Conditions for Our Featured Direct-Summation $N$-body Models

\begin{tabular}{lcccclc}
\hline \hline Model & $\mathcal{N}_{\star}$ & $\mathcal{M}_{\text {bin }} / M_{\odot}$ & $\rho_{0}\left(M_{\odot} / \mathrm{pc}^{3}\right)$ & $W_{0}$ & IMF & $a_{0}(\mathrm{pc})$ \\
\hline$A$ & 30002 & $300+300$ & $6 \times 10^{4}$ & 6 & Single & 0.01 \\
$B$ & 30002 & $1000+1000$ & $2.4 \times 10^{3}$ & 6 & Single & 0.01 \\
$C$ & 30002 & $300+300$ & $2.6 \times 10^{3}$ & 6 & Kroupa & 0.1 \\
$D$ & 30002 & $300+300$ & $6 \times 10^{4}$ & 6 & Single & 0.1 \\
$E$ & 128002 & $300+300$ & $10^{5}$ & 6 & Single & 0.3 \\
$F$ & 128002 & $1900+380$ & $10^{5}$ & 6 & Kroupa & 0.1 \\
\hline
\end{tabular}

Notes. $\mathcal{N}_{\star}$ is the number of stellar particles used. The mass of the binary, normalized to solar masses, is given in the third column, $\rho_{0}$ is the initial mass density at a distance of $0.1 \mathrm{pc}, W_{0}$ is the King parameter (King 1966). All cases are single mass but for Models $\mathrm{C}$ and $\mathrm{F}$, in which we have a mass function, specifically a $5 \mathrm{Myr}$ evolved Kroupa initial mass function (IMF) of masses 0.2 , $0.5,50$, and exponents 1.3 and 2.3 (Kroupa 2001). The seventh column shows the initial semimajor axis of the binary in pc.

Each card has a peak performance of 130 Gflops (Fukushige et al. 2005), so that a single node is comparable to a cluster of $\sim 100$ individual CPUs working in parallel.

Table 1 gives the initial conditions for the different simulations that we feature. We ran six cases with varying number densities and concentrations, of which two had a Kroupa (2001) mass function instead of single-mass stars. The IMBHs have equal mass except in simulation $F$, which has a mass ratio of 5. In our simulations the individual time steps led to fractional energy errors that were always less than $10^{-4}$ per $N$-body unit of time and, globally, the total energy error of the cluster (i.e., the accumulated error in the integration of all particles) is $0.015 \%$ in the case of our fiducial Model A.

\subsection{Evolution of the Binary: Gravitational Radiation Versus Dynamics}

Our approach is to evolve the cluster up to $\sim 50-70$ Myr using the direct NBODY4 code with a central bound binary. As we discuss below, we can see in Figure 1 that the stellar cluster experiences a very moderate expansion during the hardening of the IMBHs. Once the IMBHs are hard enough relative to each other they can be treated as an isolated binary that interacts occasionally with a passing star. We note that although the subsequent evolution of the binary will not be identical to that from the $N$-body runs, due to the stochastic nature of the encounters, the general development is similar. Such interactions tend to increase the binding energy of the binary, hence shrinking its semimajor axis. The eccentricity is also changed, both by Newtonian three-body interactions (which can increase or decrease the eccentricity; see Sesana et al. (2007) for a recent treatment) and by gravitational radiation, which circularizes the binary. The combination of the two determines the eccentricity of the binary when it enters the frequency range of LISA.

In Figure 2, we show the inspiral of the binary for Models A and $\mathrm{C}$. The irregular lines correspond to the $N$-body simulations. We take the last point of these evolutions and the number density of field stars as input to relativistic scattering experiments which we performed following Gültekin et al. (2006). The equations of motion we use for the three-body encounters include relativistic precession to first post-Newtonian order, as well as radiation reaction caused by gravitational waves. Between encounters, we evolve the semimajor axis and eccentricity of the IMBH binary using the Peters quadrupolar formulae (Peters 1964). The stars that interact with the binary are sent with a velocity of $v=$ $10 \mathrm{~km} \mathrm{~s}^{-1}$ at infinity, typical of cluster velocity dispersions. The interaction time is drawn from an exponential distribution with a mean time of $\tau=(n \Sigma v)^{-1}$, where $n$ is the stellar number density (taken from the $N$-body simulations) and $\Sigma$ is the scattering cross section including gravitational focusing. The typical region in which the IMBH binary wanders is larger than its radius of influence, hence there is no loss cone as there is for supermassive black holes.

We ran four sets of 40 simulations, two sets starting at large separations with zero eccentricity that established agreement with the direct-summation $N$-body simulations and two sets at the endpoints of Models A and B. In none of these runs was the binary itself ejected from the cluster, as was expected given its large mass. For the Model A endpoint run (binary mass $600 M_{\odot}$, initial semimajor axis $a_{0}=10 \mathrm{AU}$, and initial eccentricity $e_{0}=0.6$ ), the eccentricity was $e_{\text {LISA }}=0.30 \pm 0.10$ when the gravitational wave frequency (equal to twice the orbital frequency) was at the $10^{-4} \mathrm{~Hz}$ low end of the LISA band; for the Model B endpoint run (binary mass $2000 M_{\odot}$, initial semimajor axis $a_{0}=400 \mathrm{AU}$, and initial eccentricity $e_{0}=0.55$ ) we found $e_{\text {LISA }}=0.24 \pm 0.05$. We show the envelope of the Model B endpoint runs in the left panel of Figure 3. We also did scattering experiments corresponding to the endpoint of Model C, which had a Kroupa mass function. The results are shown in the right panel of Figure 3. Compared with the single-mass runs we see a considerably greater variance in the eccentricity as a function of the semimajor axis, and although the range of eccentricities in the LISA band $e_{\text {LISA }}=0.11 \pm 0.11$ overlaps those in the singlemass runs there are also a number of cases in which the binary is nearly circular by the time the gravitational wave frequency reaches $10^{-4} \mathrm{~Hz}$. This could be a general feature of scattering interactions when there is a broad mass function, but we have not performed enough runs to determine this with confidence.

\section{DISCUSSION AND CONCLUSIONS}

We have addressed the inspiral of two massive black holes in a single young stellar cluster. Our three main results are: (1) the cluster itself experiences only mild structural changes as a result of the inspiral; (2) the coalescence takes a short enough time (typically $<100 \mathrm{Myr}$ ) that mergers occur close to the time of formation of the cluster; and (3) there is a significant residual 

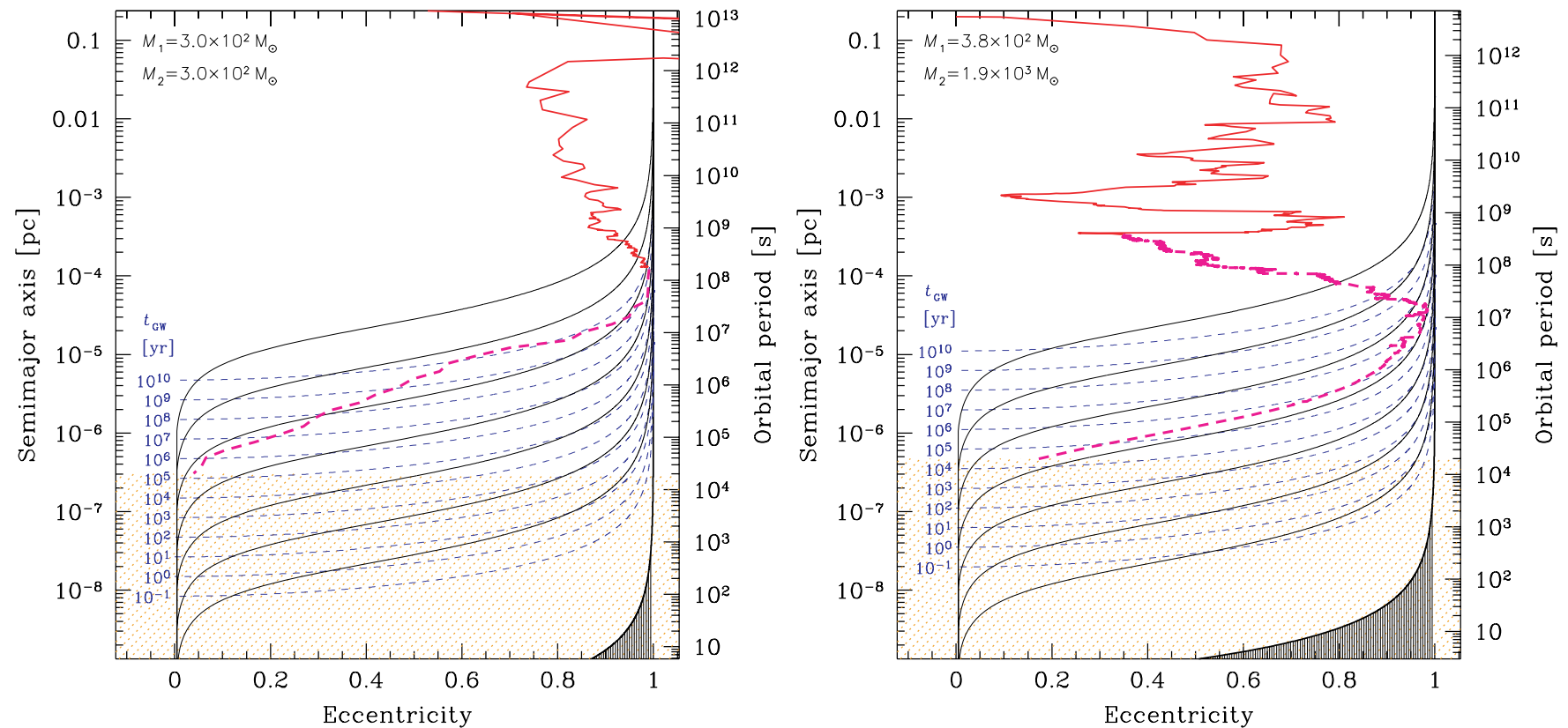

Figure 2. Left panel: inspiral of the IMBH binary of Model E followed in the eccentricity-semimajor axis plane. The irregular line shows the results of the $N$-body simulation. The smooth black solid curves are the estimated trajectories due to gravitational wave emission following the approximation of Peters (1964), and the dashed curves show the corresponding inspiral timescale, $t_{\mathrm{GW}}$. The dark dashed area depicts the region of unstable orbits. The lightly shaded area corresponds to the phase in the evolution in which the $n=2$ harmonic of the gravitational wave signal is in the LISA band. The dashed irregular lines starting after the last point of the results of the $\mathrm{N}$-body simulation (in the color version depicted in magenta) are the results from the scattering experiments. See the text for further details. Near the beginning the eccentricity temporarily exceeds unity because the black holes are not yet bound to each other. Right panel: same for Model F, which is one case in which we initially have a Kroupa IMF (see Table 1). When a star with high mass interacts with the binary, the eccentricity change is substantial. The eccentricity therefore wanders up and down, and when it becomes large enough the binary has a greater chance to spiral together by gravitational radiation.
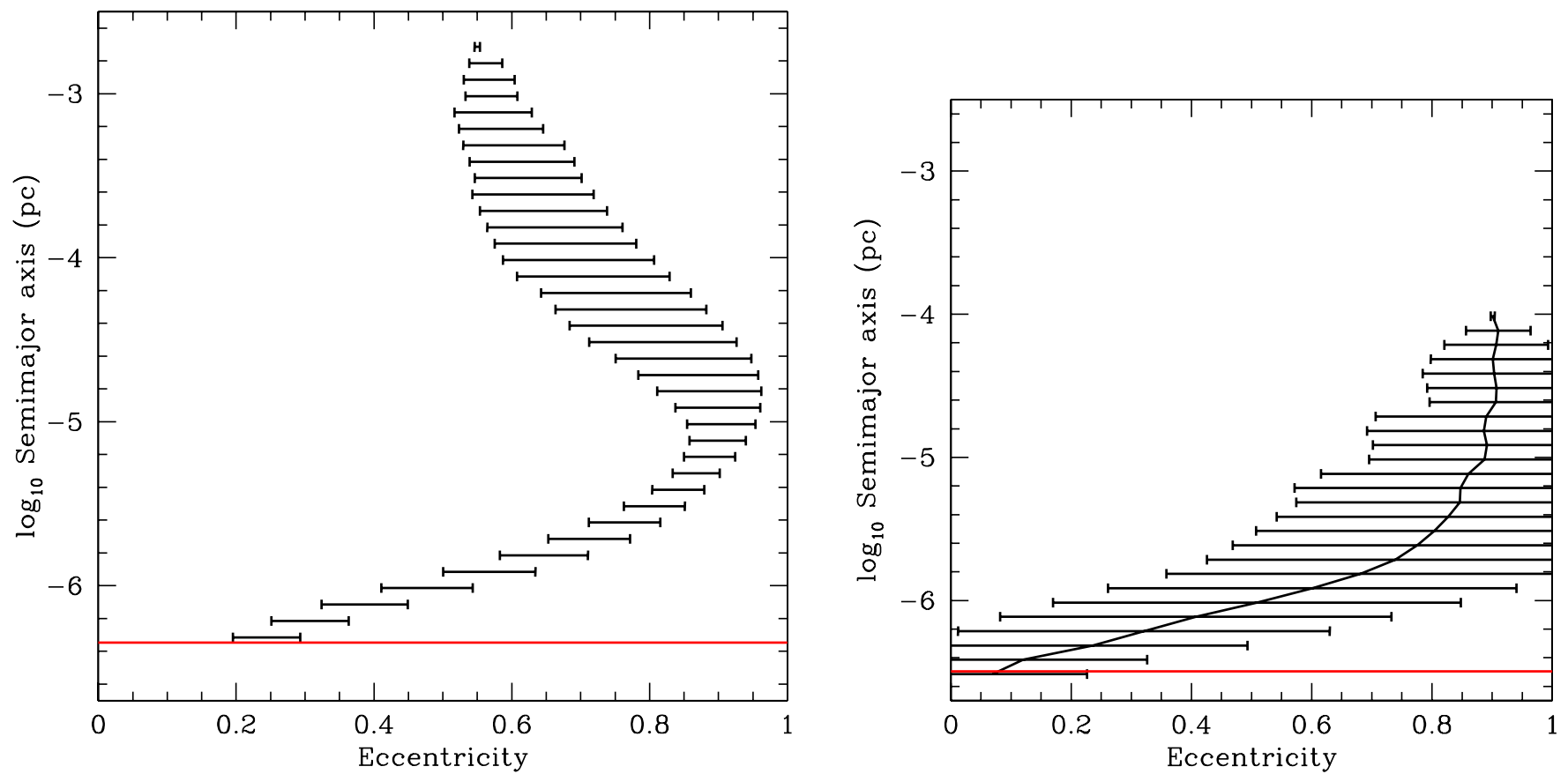

Figure 3. Left panel: average evolution of eccentricity as a function of semimajor axis. This figure shows the results of 40 three-body scattering experiments, starting with IMBH masses of $10^{3} M_{\odot}$ each at an initial semimajor axis of $400 \mathrm{AU}$ and an initial eccentricity of 0.55 , corresponding to the endpoint of Model B. We see the $-1 \sigma$ to $+1 \sigma$ ranges of eccentricity. The horizontal line shows a semimajor axis of $0.093 \mathrm{AU}$, which is where the orbital frequency is $5 \times 10^{-5} \mathrm{~Hz}$ and thus the dominant gravitational wave frequency is $1 \mathrm{e}-4 \mathrm{~Hz}$. Right panel: similar but assuming a Kroupa IMF and using the endpoint of Model C. There were no ejections of the binary from the cluster, which was assumed to have an escape speed of $50 \mathrm{~km} \mathrm{~s}^{-1}$. The horizontal line is lower than in the left panel because the binary mass is less.

eccentricity by the time the binary enters the LISA band. We now discuss these conclusions in order.

The stability of clusters against IMBH mergers is consistent with analytic expectations even though the binding energy of the IMBH binary can exceed the total binding energy of the cluster by a significant factor. To see this, consider a circular IMBH binary of component masses $m_{1}$ and $m_{2} \leqslant m_{1}$, with total mass $M_{12}=m_{1}+m_{2}$ and reduced mass $\mu=m_{1} m_{2} / M_{12}$. As 
shown by Quinlan (1996), a star with low speed at infinity that interacts with the binary will typically be ejected with a speed $v_{\mathrm{ej}} \approx 0.85 \sqrt{m_{2} / M_{12}} V_{\text {orb }}$, where $V_{\text {orb }}$ is the relative speed of the two objects. For equal masses $m_{1}=m_{2}$, this is $v_{\mathrm{ej}} \approx 0.6 V_{\text {bin }}$. Suppose now that the cluster has an escape speed $v_{\text {esc }}$. If $v_{\text {ej }}<v_{\text {esc }}$ then the star will be retained and share its kinetic energy with the cluster. Otherwise, the star will be ejected from the cluster without depositing significant energy, because the dynamical time of escape is much less than the relaxation time (which is the time required for the star to give up energy). The binding energy of the IMBH binary when $v_{\text {ej }}=v_{\text {esc }}$ will be $E_{\text {bin }}=\frac{1}{2} \mu V_{\text {orb }}^{2} \approx \frac{1}{2} \mu\left(v_{\text {esc }} / 0.85\right)^{2}\left(M_{12} / m_{2}\right) \approx 0.7 m_{1} v_{\mathrm{esc}}^{2}$. In comparison, if the cluster has a three-dimensional velocity dispersion $\sigma_{3 \mathrm{D}}$ and a mass $M_{\mathrm{cl}}$, the binding energy of the cluster is $E_{\mathrm{cl}} \approx \frac{1}{2} M_{\mathrm{cl}} \sigma_{3 \mathrm{D}}^{2}$. The ratio is then $E_{\mathrm{bin}} / E_{\mathrm{cl}} \approx$ $\left(m_{1} / M_{\mathrm{cl}}\right)\left(v_{\mathrm{esc}} / \sigma_{3 \mathrm{D}}\right)^{2}$. Typically $v_{\mathrm{esc}} \sim 2-3 \times \sigma_{3 \mathrm{D}}$, so only if the larger black hole mass is $m_{1}>0.1-0.2 M_{\mathrm{cl}}$ could the release of energy unbind the cluster. We also note that subsequent to this point, the loss of mass from stars being thrown out would also soften the cluster. However, since typically, interaction with of order the binary mass changes the semimajor axis by a factor of $\sim 2$, just $\sim 10 M_{12}$ in stars will shrink the binary by enough of a factor to produce coalescence. Therefore, as verified by our numerical simulations, hardening of an IMBH binary has only a minor effect on the cluster.

For the time to merger, we note that hardening from large separations to a few hundred AU takes $\sim 50 \mathrm{Myr}$, based on our simulations. Our three-body runs then indicate that the total time from that point to merger is virtually always less than $10 \mathrm{Myr}$, meaning that conservatively the total time from formation to merger is less than $10^{8} \mathrm{yr}$. This is significantly shorter than the age of the universe. One consequence of this is that if star formation in massive clusters was more common at redshift $z \sim 1$ than is now, and if binary IMBH formation was also thus more common, then LISA observations of IMBH mergers will serve as a unique snapshot of star formation as well as of cluster dynamics (see also, Fregeau et al. 2006).

Figure 3 shows that the eccentricity of the binary will be in the range $\sim 0.1-0.3$ when the dominant gravitational wave frequency is of $10^{-4} \mathrm{~Hz}$. Consistent with Quinlan (1996), we find that the eccentricity does not undergo a random walk, but instead tends to higher eccentricities when the binary is hard but before gravitational radiation circularization is important. As discussed in Section 4 of Amaro-Seoane \& Freitag (2006), a residual eccentricity will induce a difference in the phase evolution of the second harmonic compared to a circular orbit, even if it is as small as 0.07, as Amaro-Seoane \& Freitag (2006) found. In our case, if we use an eccentricity $e_{10^{-4} \mathrm{~Hz}}=0.3$ in Equation (4) of Amaro-Seoane \& Freitag (2006), we find that the accumulated phase shift $\Delta \Psi_{\mathrm{e}} \geqslant 2 \pi$ if observations cover a time of at least

$$
t_{\mathrm{mrg}} \sim 0.012 \cdot(1+z)^{2} \mathrm{yr}
$$

before merger, where $z$ is the redshift. If we set $z=1$, then we have to cover a time $t_{\mathrm{mrg}}=17$ days before merger. This means that if we are able to observe the system during that period of time before the final coalescence, we will recover enough information to determine that the orbit is not circular. On the other hand, if we use a residual eccentricity of 0.07 , as in Amaro-Seoane \& Freitag (2006), we would need 3-4 years of observation for a $300+300 M_{\odot}$ binary before merger.

In conclusion, if young massive clusters form binary IMBHs then they will be strong and moderately eccentric LISA sources that could serve as unique signposts of clustered star formation. The nonzero residual eccentricity has an impact on the detection of such sources, since it is generally assumed that an equalmass massive binary will have zero eccentricity when entering the LISA band. Our results show that in our scenario $e$ is nonnegligible for certain cases - though for some other models it is very low but detectable; notably, cases $C$ and $F$, which are the only models in which we have a mass fraction and thus, they are the more realistic ones. The process of formation must of course be studied carefully from the standpoints of stellar dynamics and merger product evolution, but if binary IMBHs can form then their mergers are promising sources for future LISA detections.

We thank Vanessa Lauburg for valuable discussions. We are also indebted to Yuri Levin, Ed Porter, Matt Benacquista, Jonathan Gair, and Stas Babak for enlightening conversations. The authors are grateful to the Aspen Center for Physics for its hospitality during part of this project. P.A.S.'s work was partially supported by the MEC (Ministerio de Educación y Ciencia) at the Institut de Ciències de l'Espai (IEEC/CSIC) and the DLR (Deutsches Zentrum für Luft- und Raumfahrt) at the Max-Planck Institut für Gravitationsphysik (Albert EinsteinInstitut, AEI). M.C.M. appreciates support from NASA under ATFP grant NNX08AH29G. The work of M.F. is funded through the PPARC rolling grant at the Institute of Astronomy (IoA) in Cambridge. The simulations have been performed at the GRAPE cluster TUFFSTEIN of the AEI.

\section{REFERENCES}

Aarseth, S. J. 1999, PASP, 111, 1333

Aarseth, S. J. 2003, Gravitational N-Body Simulations, (Cambridge: Cambridge Univ. Press)

Amaro-Seoane, P., \& Freitag, M. 2006, ApJ, Lett., 653, L53

Belkus, H., van Bever, J., \& Vanbeveren, D. 2007, ApJ, 659, 1756

Fregeau, J. M., Larson, S. L., Miller, M. C., O'Shaughnessy, R., \& Rasio, F. A. 2006, ApJ, 646, L135

Freitag, M., Gürkan, M. A., \& Rasio, F. A. 2006, MNRAS, 368, 141

Fukushige, T., Makino, J., \& Kawai, A. 2005, PASJ, 57, 1009

Gültekin, K., Miller, M. C., \& Hamilton, D. P. 2006, ApJ, 640, 156

Gürkan, M. A., Fregeau, J. M., \& Rasio, F. A. 2006, ApJ, 640, L39

Gürkan, M. A., Freitag, M., \& Rasio, F. A. 2004, ApJ, 604, 632

King, I. R. 1966, AJ, 71, 64

Kroupa, P. 2001, MNRAS, 322, 231

Kustaanheimo, P. E., \& Stiefel, E. L. 1965, J. Reine Angew. Math., 218, 204

Miller, M. C., \& Colbert, E. J. M. 2004, Int. J. Mod. Phys. D, 13, 1

Peters, P. C. 1964, Phys. Rev., 136, 1224

Portegies Zwart, S. F., Baumgardt, H., Hut, P., Makino, J., \& McMillan, S. L. W. 2004, Nature, 428, 724

Portegies Zwart, S. F., \& McMillan, S. L. W. 2000, ApJ, Lett., 528, L17

Quinlan, G. D. 1996, New Astron., 1, 255

Sesana, A., Haardt, F., \& Madau, P. 2007, arXiv:0710.4301 